\title{
ANÁLISE COMPARATIVA DE REDES HOSPITALARES: DIFERENÇAS ENTRE O PÚBLICO - PRIVADO
}

\section{RESUMO}

O presente analisa e compara os fatores relacionais - atuação em rede, cooperação e aprendizagem relacional - de duas redes hospitalares, a rede pública FHEMIG (Fundação Hospitalar do Estado de Minas Gerais) e a rede privada da Região Metropolitana da Grande Vitória (ES). A coleta de dados foi realizada por meio de entrevistas e questionários estruturados com gestores participantes do processo decisório. Os métodos de análise utilizados foram Regressão Linear e Análise de Redes. As hipóteses utilizadas para este estudo são: (i) atuar em rede me leva a cooperar; (ii) cooperar me leva a aprender com a rede, e; (iii) aprender com a rede me leva a reforçar minha atuação. Após a análise dos dados coletados, percebe-se que o elo de cooperação entre os hospitais é muito intenso, principalmente na rede pública. Além disso, os dados inferem que as trocas entre eles funcionam como relações colaborativas, visando à manutenção do sistema de saúde a partir do compartilhamento de competências e recursos.

Palavras-chave: Análise de Redes; Cooperação; Aprendizagem Relacional; Hospitais.

\section{COMPARATIVE ANALYSIS OF HOSPITAL NETWORKS: DIFFERENCES BETWEEN THE PUBLIC -}

\section{PRIVATE}

\begin{abstract}
This articlto analyzes and compares the relational factors - network performance, cooperation and relational learning two hospital networks, public FHEMIG (Hospital Foundation of Minas Gerais) and the private network of the Metropolitan Region of Vitória (ES). Data collection was conducted through interviews and structured questionnaires with participants managers in decision making. The analytical methods used were linear regression and Network Analysis. The assumptions used for this study are: (i) work in network leads me to cooperate; (ii) cooperate leads me to learn from the network, and; (iii) learn from the network leads me to enhance my performance. After analyzes the data collected, it can be seen that the link of cooperation between hospitals is very intense, especially in public. In addition, inferred data exchanges between them act as collaborative relationships, in order to maintain the health system from the expertise and resources sharing.
\end{abstract}

Keywords: Network Analysis; Cooperation; Relationship Learning; Hospitals. 


\section{ANALISIS COMPARATIVO DE REDES DE HOSPITAL: DIFERENCIAS ENTRE EL PÚBLICO -}

\section{PRIVADO}

\section{RESUMEN}

Este artículo analiza y compara los factores que relacionan - acción de la red, cooperación y aprendizaje relacional - de dos redes de hospitales, FHEMIG pública (Fundación Hospital de Minas Gerais) y la red privada de la Metropolitana de Vitória (ES ). La recolección de datos se realizó a través de entrevistas y cuestionarios estructurados, con los gestores participantes que detienen la toma de decisión. Los métodos analíticos utilizados fueron de regresión lineal y análisis de red. Las hipótesis utilizadas en este estudio son: (i) el trabajo en red me lleva a cooperar; (ii) cooperar me lleva a aprender de la red, y; (iii) aprender de la red me lleva a mejorar mi actuación. Después de analizar los datos recogidos, está claro que el vínculo de cooperación entre los hospitales es muy intenso, especialmente en la red pública. Además, los datos inferidos atestan que el cambio entre ellos actua como relación de colaboración, con el fin de mantener el sistema de salud a partir del principio de compartir los conocimientos e intercambiar los recursos.

Palabras clave: Análisis de Red. Cooperación; Aprendizaje Relacional; Hospitales.

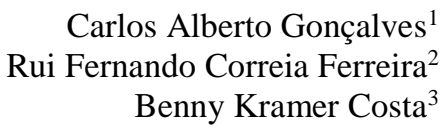

\footnotetext{
${ }^{1}$ Doutor em Administração pela Universidade de São Paulo - USP. Professor na Universidade FUMEC e Universidade Federal de Minas Gerais - UFMG. Brasil. E-mail: carlos@face.ufmg.br

2 Doutorando em Administração pela Universidade Federal Minas Gerais - UFMG. Brasil. E-mail: ruifernandof@gmail.com

${ }_{3}^{3}$ Doutor em Administração pela Universidade de São Paulo - USP. Professor, Diretor e Pesquisador do Programa de Mestrado Profissional em Administração - Gestão de Esportes da Universidade Nove de Julho - PMPA-GE/UNINOVE. Brasil. E-mail: benny@uni9.pro.br
} 


\section{INTRODUÇÃo}

O estudo de redes tem sido tratado com maior ênfase por pesquisadores em uma perspectiva interorganizacional. Alguns destes estudos que abordaram o tema de redes interorganizacionais são os estudos seminais Ahuja (2000), Gulati \& Gargiulo (1999), Owen-Smith \& Powell (2004), Phelps, Heidl, \& Wadhwa (2012), Provan \& Milward (1995), Provan; Fish \& Sydow (2007). A análise de redes interorganizacionais vem recebendo atenção relativamente recente, e crescente, de autores brasileiros dentro dos estudos da estratégia. Ao realizar uma busca no SPELL - Scientific Periodicals Electronic Library da Associação Nacional de PósGraduação e Pesquisa em Administração - ANPAD, com as palavras 'redes interorganizacionais' foram encontrados dezenas de trabalhos publicados sobre a temática.

Em um contexto competitivo globalizado, a busca pela vantagem competitiva tornou-se mais árdua e mais complexa, exigindo, por parte das empresas, níveis de serviço superiores a preços cada vez mais reduzidos (Hitt,, Ireland, \& Hoskisson, 2008). Como resposta a esse desafio, surgem novas estruturas organizacionais, que estão ligadas à constituição de novas formas de relacionamento entre empresas (Amato Neto, 2000). Para Peci (1999), no atual ambiente competitivo, as empresas devem se concentrar nas suas competências básicas e estabelecer parcerias com outras organizações por meio da formação de redes organizacionais. Castells (1999) concorda com a importância da estrutura em rede e afirma que as redes são e serão os componentes fundamentais das organizações e que tal estrutura será a forma predominante de concorrência na nova economia global. Esse autor destaca ainda que as organizações tradicionais, ao operarem de forma individualizada, enfrentarão dificuldades quanto aos seus novos desafios.

Um dos segmentos mais complexos de analisar as relações em rede é o setor de saúde. Como argumenta Daft (2006), o hospital é uma empresa de serviços, que pode ser classificada a partir das cinco dimensões propostas pelo autor: (1) produção e consumo simultâneos; (2) produtos personalizados; (3) participação dos pacientes nos processos de produção; (4) produto intangível; e, (5) ênfase em pessoas. Estas características tornam a Gestão Hospitalar complexa e difícil mensuração em seus resultados. Para mais, a realidade brasileira envolve provedores de serviços em saúde públicos e privados em uma proposta de atendimento universal, tornando o processo gerencial complexo, não só da perspectiva financeira, mas também da política e organizacional. Uma das formas de superar as dificuldades gerenciais e contingenciais é a atuação em rede.

Como concluem De Borba \& Neto (2008) existe uma grande preocupação com o uso e a construção do conhecimento para a prática de gestão na área de saúde. Essa necessidade de mudança comportamental nas práticas de saúde, buscando o conhecimento coletivo e uma ênfase na explicitação dos conhecimentos para a organização. Portanto, analisar as formas de cooperação e aprendizagem das organizações hospitalares em rede torna-se necessário para proposição de novas práticas na gestão destas organizações. O modo como os estrategistas dos serviços de saúde atuam em rede, pode ser observado para melhor compreender os fenômenos organizacionais de competição, cooperação e aprendizado relacional. Esse entendimento é relevante para a identificação dos fatores chave ao analisar o comportamento em redes.

Objetiva-se compreender Como os fatores relacionais - (i) Atuação em Rede, como as empresas se comunicam, trocam informações, participam de eventos em conjunto; (ii) Cooperação, como as empresas trocam recursos e informações estratégicas, e; (iii) Aprendizagem Relacional, como as empresas aprendem umas com as outras e produzem conhecimento - se comportam em uma rede pública, localizada em Belo Horizonte/MG e região Metropolitana e uma rede privada, na região metropolitana da grande Vitória/ES. Para isso, este estudo pretende analisar e comparar os fatores relacionais Atuação em Rede, Cooperação e Aprendizagem Relacional nas duas redes hospitalares, público e privado, por meio da Análise de Redes Sociais e Análise de Regressão Linear. Logo seguem os itens aprofundados nas bases teóricas e empíricas deste artigo.

\section{REFERENCIAL TEÓRICO}

O significado de "rede" pode variar conforme o campo de conhecimento, o contexto e a aplicação. Independente do campo de conhecimento, rede é compreendido como um conjunto de nós interconectados (Castells, 1999). Este estudo apoia-se na concepção de redes interorganizacionais como constelações de organizações que se reúnem para estabelecer contratos ou acordos sociais (Barringer \& Harrison, 2000). Por ser mais abrangente, a definição de Barringer e Harrison (2000) é mais coerente com a abordagem epistemológica adotada, baseada em aspectos sociais, para a compreensão e análise do fenômeno das redes interorganizacionais.

O conceito "rede", por si só, é uma abordagem abstrata no que se refere a um conjunto de "agentes" conectados por relacionamentos interorganizacionais. $\mathrm{Na}$ teoria das organizações, o emprego do conceito de rede torna-se aplicável quando demonstra modos de coordenação, diferenciação e integração entre os participantes da rede que atuam de forma especializada, tendo em vista que, nas relações econômicas, as organizações são precedidas por relações sociais 
Análise Comparativa de Redes Hospitalares: Diferenças entre o Público - Privado

(Castells, 1999). O estudo das redes tornou-se um ponto de confluência de várias disciplinas e de distintas abordagens e um espaço fecundo de interação e sinergia entre conceitos teóricos, metodologias e resultados empíricos (Vasconcellos \& Lopes, 2010). Alguns conceitos da Analise de Redes Sociais segue apresentado pelo Quadro 1.

\begin{tabular}{|c|l|}
\hline Ator & É cada indivíduo, setor ou departamento que está interligado à rede. \\
\hline Ligações & São representações gráficas de linhas que conectam os pontos (atores). \\
\hline Subgrupos & $\begin{array}{l}\text { Subconjuntos de atores de determinada rede. A formação destes subconjuntos pode estar } \\
\text { relacionada com posição hierárquica, localização, afinidade, idade, escolaridade, sexo. Quando } \\
\text { envolvem ligações entre dois atores são "díades" e quando envolvem três atores são "tríades". }\end{array}$ \\
\hline Relação & É um tipo específico de lidações de um determinado grupo. \\
\hline Tamanho & É a quantidade de conexões existentes entre os atores de uma rede. \\
\hline $\begin{array}{c}\text { Distância } \\
\text { Geodésica }\end{array}$ & É o caminho mais curto entre dois atores de uma rede. \\
\hline Coesão & $\begin{array}{l}\text { Trata-se do forte relacionamento entre atores de uma rede, formando subgrupos em virtude de } \\
\text { vínculos estabelecidos por afinidades. Este campo de estudo possui forte apelo na correlação entre } \\
\text { a coesão e padrões de comportamento destes subgrupos. }\end{array}$ \\
\hline Reciprocidade & $\begin{array}{l}\text { As ligações entre os atores podem ser analisadas quanto ao sentido (representadas por setas). } \\
\text { Nestes casos, a ligação é recíproca quando flui nos dois sentidos. }\end{array}$ \\
\hline Posição & $\begin{array}{l}\text { Diz respeito aos indivíduos que estão, de forma semelhante, envolvidos em redes de relação, logo, } \\
\text { potencialmente, intercambiáveis sob a ótica da análise sociológica. }\end{array}$ \\
\hline Papel & Diz respeito aos padrões de relações obtidas entre atores ou posições. \\
\hline
\end{tabular}

Quadro 1 - Conceitos e medidas para Análise de Rede.

Fonte: Azevedo \& Rodriguez, 2010; Lago Júnior, 2005

Ao aprofundar sobre tipologia de rede, Lazzarini (2008) classifica as redes como horizontais e verticais, e afirmando que é muito raro, em contexto interorganizacional, encontrar laços baseados exclusivamente em uma dessas características. Para o autor, grande parte das redes existentes é formada por um conjunto complexo desses laços. Outro fenômeno da análise de redes são buracos estruturais apresentados por Burt (2009), em que redireciona a atenção para a forma ou topologia da rede do ego de um ator. Especificamente, Burt (2009) equipara o desempenho na rede do ator com a falta de laços entre os atores de um nó, uma condição que ele nomeia furos estruturais. Ele argumenta que a abrangência de furos estruturais fornece o real mecanismo para mensurar os laços fracos (Borgatti \& Foster, 2003).

Para Porter (1998), o conceito "redes" extrapola o âmbito da teoria organizacional e é definido como o método organizacional de atividades econômicas por meio da coordenação e da cooperação entre as empresas. Já Lazzarini (2008), define redes como um conjunto de organizações interligadas por meio de relações que se dão de diversas formas possíveis. Em perspectiva cooperativista, Jarillo (1988) explica "redes" como um acordo de longo prazo, com propósitos bem definidos, entre empresas distintas, que têm um relacionamento em comum. Dessa maneira, é possível desenvolver ou sustentar uma vantagem competitiva diante das demais empresas que não participam da rede. As redes são, então, estruturas formadas a partir da definição de papeis, atribuições e relações entre seus atores, o que permite a flexibilização do funcionamento da rede através de relações de cooperação. Porém, os autores afirmam que esses fatores de atuação em rede não eliminam, necessariamente, os conflitos e a competição.

$\mathrm{O}$ ambiente externo das organizações está em constante alteração, em meio a um cenário de incerteza. A empresa hierarquizada e de estrutura engessada, não consegue adaptar-se frente as intensas mudanças econômicas, tecnológicas e institucionais. Neste contexto, surgem novas formas organizacionais e atuar em rede é uma das possibilidades. As redes organizacionais nascem em consequência da coexistência de fatores como: 1) resposta às mudanças ambientais, que conduzem à necessidade de interdependência; 2) incapacidade das empresas focadas em escala integrada verticalmente e de pequenas empresas isoladas sobreviverem em ambiente altamente mutável; e 3) demanda por recursos especializados (Peci, 1999).

A ideia de redes sociais agrega à teoria de estratégia a compreensão de que as organizações agem no interior de um sistema de relações concretas e em permanente desenvolvimento, em redes interorganizacionais. Assim, a noção de redes, utilizada inicialmente por uma sociologia relacional, vem sendo aplicada "aos arranjos organizacionais flexíveis e plurais que se formam no setor econômico, político ou sociais" (Migueletto, 2001, p. 7).

Em um estudo bibliométrico realizado por Balestrin, Verschoore e Junior (2010) com dados de 2000 a 2006 do campo de redes interorganizacionais no Brasil, identificou mais de uma centena de trabalhos. Como argumenta os autores, os resultados demonstram o crescimento do campo no Brasil que se observa em 
Análise Comparativa de Redes Hospitalares: Diferenças entre o Público - Privado

contínua expansão e robustez. Conforme os autores concluem no estudo as redes horizontais (simétricas), formadas por múltiplas organizações de um mesmo elo da cadeia produtiva, são o principal interesse das pesquisas, mas não falam sobre comparações estruturais de diferentes redes do mesmo seguimento.

Corroborando com Balestrin, Verschoore e Junior (2010) este trabalho possui orientação predominante de teorias de estratégia, dependência de recursos, redes sociais e institucionais. Como premissa deste trabalho apresentado por Balestrin, Verschoore e Junior (2010), os antecedentes que levam as organizações Atuar em Redes de cooperação com maior é a necessidade de acesso a recursos materiais e imateriais, além da congruência de objetivos. Para manutenção e convergência da rede, os autores defendem que o fator Aprendizagem, é um dos resultados ou ganhos proporcionados pelas redes de cooperação com maior interesse nos estudos brasileiros. Os construtos teóricos trabalhados como fatores relacionais deste trabalho estão apresentados a seguir.

\subsection{Modelo hipotético proposto}

\subsubsection{Atuação em rede}

Para Borgatti \& Foster, (2003) o ator é normalmente visto como um agente racional, ativo que explora a sua posição na rede, a fim de maximizar o ganho. A posição do ator na rede é apresentada por uma representação abstrata de laços desejáveis. Os benefícios para o ator é ligada a posição topológica na estrutura da rede, compreendendo a possibilidade de alavancar as estruturas relacionais da empresa. Assim, o comportamento de cada ator é interpretado a partir de padrões de vínculos interacionais e morfológicos em sua rede social. Em uma perspectiva topológica as interdependências causais e interacionais em configurações sociais devem ser consideradas. As regularidades dos caminhos entre atores dos grafos dividem o campo social em várias regiões. Desta forma, as oportunidades para atores moverem-se no mundo de redes sociais são determinadas por fronteiras das regiões em que estão alocados. As restrições impostas pelas fronteiras são as forças que determinam o comportamento do grupo (Scott, 2012).

As atuações em rede podem ser explícitas ou implícitas e tem sido observadas em diversos tipos de segmentos econômicos. As explícitas evolvem acordos formais, conhecidos publicamente e de caráter multilateral. Já nas implícitas os acordos baseiam-se em estrutura bilateral entre grupos informais. Nestas, há tendência de maior interação entre membros que mantêm maior ligação bilateral em comparação a empresas externas a rede (Lazzarini, 2008).

Para apresentação do modelo hipotético utilizado neste trabalho, considera-se atuar em rede participar de forma institucionalizada ou não, por meio de contatos ou reuniões em conjunto, sem necessidade de cooperar. Como paradoxo das teorias de estratégia que explicam o comportamento em rede, os fatores relacionais se embasam em uma díade de competir/cooperar, em que em situações de grande complexidade pode-se cooperar em determinado contexto e competir em outro (Vasconcellos \& Lopes, 2010). Compreende-se cooperação como troca de recursos, informações e conhecimento. A partir disso, tem-se a primeira hipótese deste estudo: (H1o) atuar em rede me leva a cooperar.

\subsubsection{Cooperação}

Para Smith, Carroll e Ashford (1995), muitas definições de cooperação enfocam processos pelos quais agentes se unem, interagem e formam relações para ganhos ou benefícios mútuos. Já Ring e Van de Ven (1994) definem a cooperação de forma dinâmica, incluindo a disposição dos agentes em continuar as relações de cooperação, que são mecanismos socialmente construídos para a ação coletiva, a qual é continuamente reestruturada por ações e interpretações simbólicas das partes.

Amato Neto (2000) afirma que uma rede de cooperação entre empresas une organizações que antes eram fechadas e independentes. Empresas que cooperam entre si têm em comum os seguintes pontos: proximidade geográfica e pertencem a um mesmo segmento de mercado. Essas organizações que atuam de forma cooperativa conseguem coordenar-se para melhorarem suas condições competitivas no mercado, por meio de estratégias comuns aos envolvidos na rede.

Verschoore (2004) afirma que as redes de cooperação são formadas por um grupo de empresas com objetivos em comum, que estão formalmente relacionadas e que a cooperação não possui prazo para o seu termino, sendo uma relação duradoura. Assim, tem-se que o desenvolvimento de ações conjuntas entre empresas que visam objetivos em comum é a base do conceito de cooperação entre empresas. Além disso, considera-se que uma premissa das teorias de estratégia relacionadas com aprendizagem e redes é que cooperar tanto troca como desenvolve conhecimento tácito entre atores da rede, possibilitando o surgimento de vantagens competitivas (Prahalad \& Hamel, 2005). Tem-se a segunda hipótese: (H2o) cooperar me leva a aprender.

\subsubsection{Aprendizagem relacional}

Como afirma Borgatti \& Foster, (2003) o mantra atual é que a criação e utilização do conhecimento são fundamentalmente humanas e acima de tudo processos sociais. Fleury e Fleury (1997) afirma que é pelo processo de aprendizagem e gestão do conhecimento que as organizações podem desenvolver competências necessárias para obterem vantagem competitiva, ou preservarem a sobrevivência no mercado. Atualmente, privilegia-se o integrado, em detrimento do que fragmenta e isola organizações, em 
que o mais relevante é a integração de conhecimentos, que eleva a aprendizagem das organizações que atuam integradas, tanto em relação aos indivíduos, quanto às relações organizacionais (Fleury \& Fleury, 1997).

Para Senge (2009), à medida que o mundo se torna mais interligado e os negócios mais complexos e dinâmicos, o trabalho torna-se cada vez mais ligado ao aprendizado. Não basta que poucas pessoas aprendam e detenham o conhecimento da organização. É preciso que todos os níveis e departamentos da empresa envolvam-se na aprendizagem, de modo a incentivar o pensamento sistêmico, em que a totalidade pode ser maior que a soma das suas partes.

Independente da transformação no aprendizado organizacional ao longo do tempo, observa-se que o conhecimento tornou-se importante fonte de vantagem competitiva (Prahalad \& Hamel, 2005; Nonaka \& Takeuchi, 1997) e que o principal fator de produtividade e competitividade das organizações está em gerar, processar e transformar conhecimento em ativo econômico (Balestrin \& Fayard, 2003). Tem-se que o conhecimento, mais que o capital ou o trabalho, é o recurso mais relevante da sociedade pós-capitalista capaz de definir a posição competitiva das empresas (Druker, 1999). A aprendizagem e produção de conhecimento nas organizações pode ser reforçada quando se atua em rede (Drucker, 1999).

Para Borgatti \& Foster, (2003) o termo gestão do conhecimento pode em breve desaparecer à medida que os atores se esforçam pouco para utilizar as soluções tecnológicas para ajudar as organizações a armazenar, compartilhar e criar novos conhecimentos, ou seja, se os atores percebem que não estão aprendendo, pode-se criar buracos estruturais. Diante disso, considera-se relevante a seguinte hipótese: (H3o) apreender com a rede me leva reforçar a atuação em rede.

Figura 1 - Modelo hipotético proposto

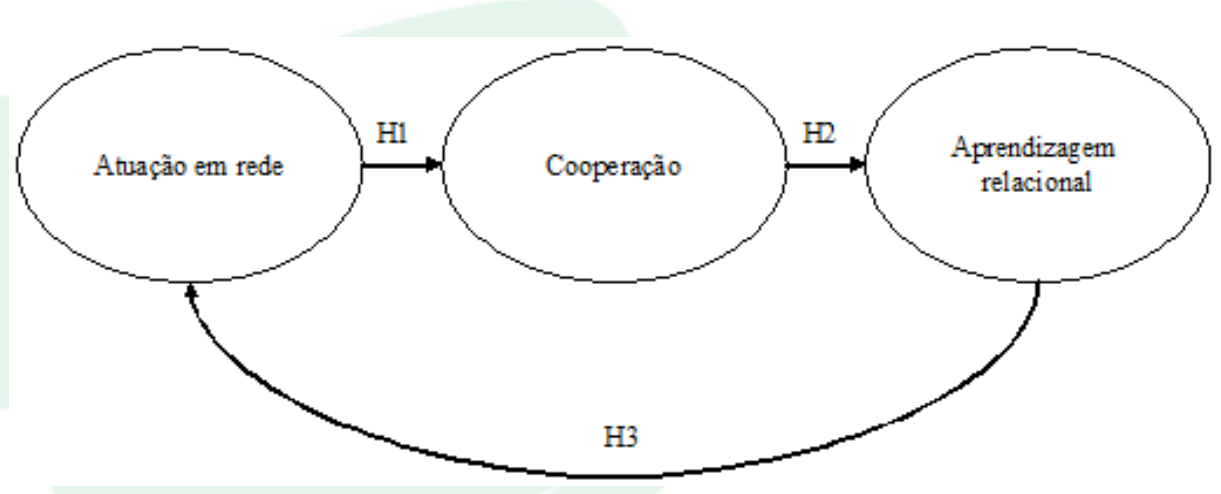

Fonte: Elaborado pelos autores.

\subsection{Hospitais públicos e privados}

Com a evolução dos serviços em saúde ampliou a profissionalização da atividade hospitalar e suas funções variadas, surgindo à necessidade da gestão administrativa, médicos, enfermeiros e outras profissões relacionadas, aumentando a complexidade das instituições hospitalares (Glouberman \& Mintzberg, 2001; Porter \& Teisberg, 2007).

Daft (2006) descreve o hospital como uma empresa de serviços, que pode ser classificada a partir das cinco dimensões propostas pelo autor: (1) produção e consumo simultâneos: o atendimento caracteriza-se como um serviço. O paciente recebe ao mesmo tempo em que é realizado pelo médico; (2) produtos personalizados: apesar dos padrões de conduta e protocolos, que apoiam decisão médica, os pacientes recebem atendimento personalizado, pois é percebido como único para o prestador do serviço; (3) participação dos pacientes nos processos de produção: às informações dos pacientes são fundamentais para o diagnóstico do médico; (4) produto intangível: no processo de atendimento, não existe produto físico resultante. $\mathrm{O}$ produto é o próprio atendimento; e, (5) ênfase em pessoas: os serviços são realizados pelos funcionários na presença dos pacientes. Além disso, a complexidade de gestão na área hospitalar alinha-se à avaliação do hospital, como componente de um sistema de saúde mais amplo e à análise das características internas do hospital.

(A complexidade dos hospitais deriva de suas características, como: I) dificuldade para definir e medir resultados; II) grande variabilidade do trabalho desenvolvido; III) natureza emergencial das atividades; IV) pouca margem de ambiguidade e de erro aceitável; V) a alta lealdade a profissão acima da organização; VI) pouco controle organizacional ou gerencial sobre o grupo médico e VII) existência de dupla linha de autoridade, a técnica e a burocrática, gerando problemas de coordenação e responsabilidades (Shortell \& Kaluzny, 2000). Hoje os hospitais são referências na cadeia de serviços de assistência de saúde, representando dois terços dos gastos do setor e boa parte dos serviços produzidos (Santos, 1998). 
Análise Comparativa de Redes Hospitalares: Diferenças entre o Público - Privado

La Forgia e Couttolenc (2009,) afirmam que a administração dos serviços hospitalares são complexos e onerosos resultando em grandes desafios para gestão. Compreender os processos e componentes do serviço hospitalar é necessário. Ao ponto que é importante controlar os resultados e desempenhos de forma confiável e atualizada. Os hospitais no Brasil são descritos quanto ao tipo por geral e especializado. Um hospital é geral quando se destina à prestação de atendimento nas especialidades básicas, podendo dispor de urgência / emergência. O hospital especializado é destinado à prestação de assistência à saúde em uma única especialidade, podendo dispor de urgência / emergência (Brasil, 2011).

A natureza administrativa que define a vinculação administrativa dos hospitais a origem do capital social classificam-se: administração direta da saúde, administração direta de outros órgãos, administração indireta (de autarquias, fundação pública, empresa pública, organização social pública), serviço social autônomo, economia mista, sindicato, fundação privada, empresas privadas, cooperativas e entidade beneficente sem fins lucrativos (Brasil, 2011).

\section{MÉTODO}

A pesquisa é quantitativa e faz uso do instrumento metodológico de questionário para coleta de dados. Em geral consiste em questionário estruturado com métrica e representação numérica, com número significativo de respondentes, destinado a obter informações específicas dos entrevistados. O estudo é considerado transversal, pois representa um corte no tempo da realidade observada (Malhotra, 2012; Gonçalves \& Meirelles, 2004).

\subsection{População}

A população de redes é, por natureza, não probabilística. Em geral, os pesquisadores de redes estão mais focados na descoberta de relações entre parâmetros/variáveis e a base teórica do que em deduzir padrões aplicáveis a uma população de redes alheia ao objeto de estudo. Por isso, este estudo não utiliza amostra (Gonçalves \& Lebarcky \& Muylder 2015). Foram utilizados dados de 21 hospitais da rede pública FHEMIG e 21 hospitais privados da grande região de Vitória.

A Fundação Hospitalar do Estado de Minas Gerais (FHEMIG) foi criada pela Lei 7.088, em 1977, e todos os hospitais da rede são vinculados à Secretaria de Estado de Saúde de Minas Gerais. A FHEMIG, atualmente, é constituída em rede com vinte unidades hospitalares, onze localizadas na capital e as demais em Bambuí, Barbacena, Betim, Juiz de Fora, Patos de Minas, Sabará, Três Corações e Ubá. A Rede FHEMIG é uma das maiores gestoras de hospitais públicos do País e tem atuação em seis complexos assistenciais: Urgência e Emergência, Especialidades medicas, Saúde
Mental, Hospitais Gerais Reabilitação e Cuidado ao Idoso e MG Transplantes.

A RMGV atualmente possui unidades em Vitória, Vila Velha, Cariacica, Serra, Fundão e Guarapari. Estes sete municípios abrigam quase metade da população total do Espírito Santo (46\%) e 57\% da população urbana do estado. Produzem $58 \%$ da riqueza e consomem $55 \%$ da energia elétrica produzida no ES. População estimada em 1.730 .000 habitantes. Os hospitais que servem à população se distribuem de forma homogênea nos municípios de Vitória, Vila Velha, Cariacica e Serra (Espirito Santo, 2005). Dos 21 hospitais, $81 \%$ é de natureza privada lucrativa. Os demais $(19 \%)$ caracterizam-se como entidade beneficente sem fins lucrativos.

\subsection{Coleta e tratamento dos dados}

Para esta pesquisa, utilizaram-se como instrumentos e técnicas de coletada de dados questionários e entrevistas estruturadas com gestores participantes do processo decisório das unidades hospitalares. Foram entrevistadas 42 pessoas participantes ativas do processo decisório das redes analisadas, portanto, 21 diretores da FHEMIG e 21 proprietários dos Hospitais privados e filantrópicos da RMGV, entre os meses de novembro de 2012 e março de 2013. Todos os questionários aplicados foram validados. A entrevista estruturada seguia uma das abordagens para a pesquisa em redes sociais, a lista fixa. Ou seja, compilou-se uma lista de atores (ou organizações) antes da coleta de dados começar (Carrington, Scott, \& Wasserman, 2005). Assim, utilizou-se um questionário para a rede pública com os hospitais da FHEMIG, e outro com os hospitais privados do Espírito Santo, seguindo a mesma estrutura. A partir do banco de dados tabulado em Excel foram realizadas análises pelo software Ucinet. Ucinet, apesar de pago, é uma das melhores ferramentas para análise de redes, pela variedade de medidas que processa (Borgatti, 2002).

\subsection{Método de análise dos dados}

\subsubsection{Análise de redes sociais}

A abordagem da análise de redes sociais tem sua base na sociologia, psicologia social e antropologia (Freeman, 1996). Wasserman e Faust (1994) argumentam que os atores da rede, em que as ligações são observadas, são representações de indivíduos, grupos, empresas e demais grupos. O que diferencia esse tipo de estudo é a ênfase nas ligações entre os atores, e não às características individuais de cada ator.

A análise de redes possui diversas vantagens metodológicas, que compreendem desde a exploração de mapas visuais até a elaboração de modelos matemáticos. Os estudos de redes sociais utilizam design de redes egocentradas (egocentric) ou de redes inteiras (whole-network). $\mathrm{Na}$ primeira observam 
relacionamentos de um ator com outros atores, enquanto na segunda examinam conjuntos de objetos interrelacionados (Marsden, 2005). Quatro características estão na análise de redes sociais moderna (Freeman, 2004): a) análises de redes sociais são motivadas por uma visão estrutural baseada em laços vinculando atores sociais; b) são fundadas em dados empíricos sistemáticos; c) baseiam-se fortemente na imagem gráfica; e d) baseiam-se no uso de modelos matemáticos e computacionais.

Os principais tipos de informação em análise de redes são informações de atributo (atitudes, comportamentos, qualidades e características), denominadas também como "propriedades", e informações relacionais (contatos, laços e conexões que relacionam um agente a outro) (Scott, 2012). Com importantes contribuições nas áreas de Saúde, Sociologia, Psicologia, Administração Pública, Física, Estratégia, Comportamento Organizacional, Comunicação e Ciências da Computação a análise de redes tem se destacado (Provan; Fish \& Sydow, 2007). Para facilitar a interpretação dos resultados da rede, torna-se relevante a adoção de conceitos para interpretação das medidas de centralidade e coesão da rede, vide Quadro 3.

\begin{tabular}{|c|l|}
\hline Tamanho & Indica o total de atores que compõe a rede, bem como a totalidade de relações identificadas. \\
\hline Densidade & $\begin{array}{l}\text { É o quociente entre o número de ligações existentes pelo número de ligações possíveis um uma } \\
\text { determinada rede. Esta retrata a potencialidade da rede em termos de fluxo de informações, ou } \\
\text { seja, quanto maior a densidade mais intensa a troca de informações na referida rede e vice-e-versa. }\end{array}$ \\
\hline Grau de saída & $\begin{array}{l}\text { É a medida do número de ligações que um ator estabelece co outros atores desta rede, denotando } \\
\text { expansividade. }\end{array}$ \\
\hline $\begin{array}{c}\text { Grau de } \\
\text { entrada }\end{array}$ & $\begin{array}{l}\text { É a medida do número de ligações que um ator recebe de outros atores, denotando popularidade ou } \\
\text { receptividade. }\end{array}$ \\
\hline $\begin{array}{c}\text { Grau de } \\
\text { centralidade }\end{array}$ & $\begin{array}{l}\text { Calcular a centralidade de um indivíduo da rede significa analisar a posição em que este se } \\
\text { encontra em relação aos outros. A centralidade considera como medida a quantidade de elos que se } \\
\text { colocam entre eles. Esta não é uma posição fixa, nem hierárquica, mas, em se tratando de redes, a } \\
\text { centralidade de um ator pode significar poder. A posição centralizada de um indivíduo na rede } \\
\text { favorecerá o recebimento e troca de comunicação ao mesmo tempo. Entretanto, o fato de um } \\
\text { indivíduo não estar em posição central na rede não significa necessariamente que este não esteja } \\
\text { bem posicionado na rede. }\end{array}$ \\
\hline $\begin{array}{c}\text { Grau de } \\
\text { Proximidade }\end{array}$ & $\begin{array}{l}\text { Esta medida caracteriza a independência de um ator em relação ao controle dos outros. Na } \\
\text { centralidade de proximidade, considera-se que um nó é tão mais central quanto o menor caminho } \\
\text { que este necessita percorrer para alcançar outro indivíduos da rede. }\end{array}$ \\
\hline $\begin{array}{c}\text { Grau de } \\
\text { Grau de } \\
\text { reciprocidade }\end{array}$ & $\begin{array}{l}\text { Esta calcula o potencial dos elos que servem de intermediários, "ponte" na rede. Estes indivíduos } \\
\text { "ponte" são facilitadores do fluxo da informação entre os elos da rede. Um indivíduo pode não } \\
\text { estar em posição central, nem mesmo possuir contatos fortes, mas pode ser importante mediador } \\
\text { no fluxo, e direção das informações. }\end{array}$ \\
\hline considerando o total de pares que mantêm relação entre si (relações simétricas e assimétricas).
\end{tabular}

Quadro 2 - Conceitos das medidas de centralidade utilizados

Fonte: Azevedo \& Rodriguez, 2010; Lago Júnior, 2005.

\subsubsection{Análise de regressão linear}

Como o objetivo inicial deste trabalho foi comparar os fatores relacionais dentro dos contextos de rede hospitalar público e privado, propõe-se realizar uma análise de regressão linear simples para verificar o modelo proposto (Fig. 1). A análise de regressão tem como objetivo verificar relações de causa e efeito, e mensurar a significância destas relações (Hair Jr, Anderson, Tatham \& Black, 2005). Para os dados da regressão foram utilizados o grau médio de entrada e saída da metodologia de redes. Estas medidas são geradas pelo software UCINET, por meio da média de números atribuídos para cada relação, por cada ator da rede. Desta forma, possibilita-se correlacionar os graus de centralidade dos fatores Atuação em Rede, Cooperação e Aprendizagem relacional. Para isso, foi necessário avaliar os pressupostos necessários a uma análise linear de dados, utilizando-se para este cálculo o software estatístico SPSS for Windows 17.0. Pelo fato de baixa quantidade de casos compondo a amostra, foi realizada, então, a análise de regressão linear simples, para testar as hipóteses da pesquisa.

\section{RESULTADOS E DISCUSSÃO}

Para facilitar a compreensão dos graphos segue na Fig. 2 uma legenda para interpretação das relações. O tamanho dos nós está relacionado a medida de centralidade, as cores dos nós está categorizada com os resultados de cada grupo de hospitais, as cores dos laços está relacionada com a reciprocidade ou não reciprocidade do laço. Cada nó (círculo) representa 
uma organização, as setas que saem do nó representam o grau de saída, e as que indicam para o nó, o grau de entrada. Assim, observa-se a direção relacional da interação entre os nós da rede.

Figura 2 - Legenda Gráfica da Rede: Laços e Nós

\begin{tabular}{|c|c|c|c|}
\hline & \multicolumn{3}{|c|}{ Cor do laço: Relação Recíproca } \\
\hline & \multicolumn{3}{|c|}{ Cor do laço: Relação NÃO Recíproca } \\
\hline $\begin{array}{l}\text { "K-cores", que categoriza os nós pela distância } \\
\text { geodésica na rede, formando blocos de nós que possuem } \\
\text { distâncias parecidas. O tamanho dos nós definidos pelo } \\
\text { grau de centralidade "Egeinvector" em que os nós mais } \\
\text { conectados - com maior quantidade de vetores - é maior, } \\
\text { respectivamente, os que possuem menor quantidade de } \\
\text { vetores são menores. }\end{array}$ & $\begin{array}{l}\text { Cada círculo } \\
\text { representa um } \\
\text { ator ou nó. }\end{array}$ & $\begin{array}{l}\text { Quando a seta relacional } \\
\text { "sai"do nó, representa } \\
\text { um Grau de Saída } \\
\text { graficamente }\end{array}$ & $\begin{array}{l}\text { Quando a seta relacional } \\
\text { "entra"no nó, representa um } \\
\text { Grau de Entrada grafica- } \\
\text { mente }\end{array}$ \\
\hline
\end{tabular}

Fonte: Elaborado pelos autores.

Logo, seguem as análises de cada fator relacional, com suas medidas e interpretações. Para as interpretações dos dados foram utilizados os conceitos, índices e medidas apresentadas anteriormente neste trabalho. O grapho relacional atuação em rede (Fig. 3) se originou da pergunta "Com quem o hospital mantém contato e ATUA EM REDE (troca ligações, troca emails, participa de reuniões, encontra em eventos)".

Figura 3 - Atuação em rede - Privado versus Público
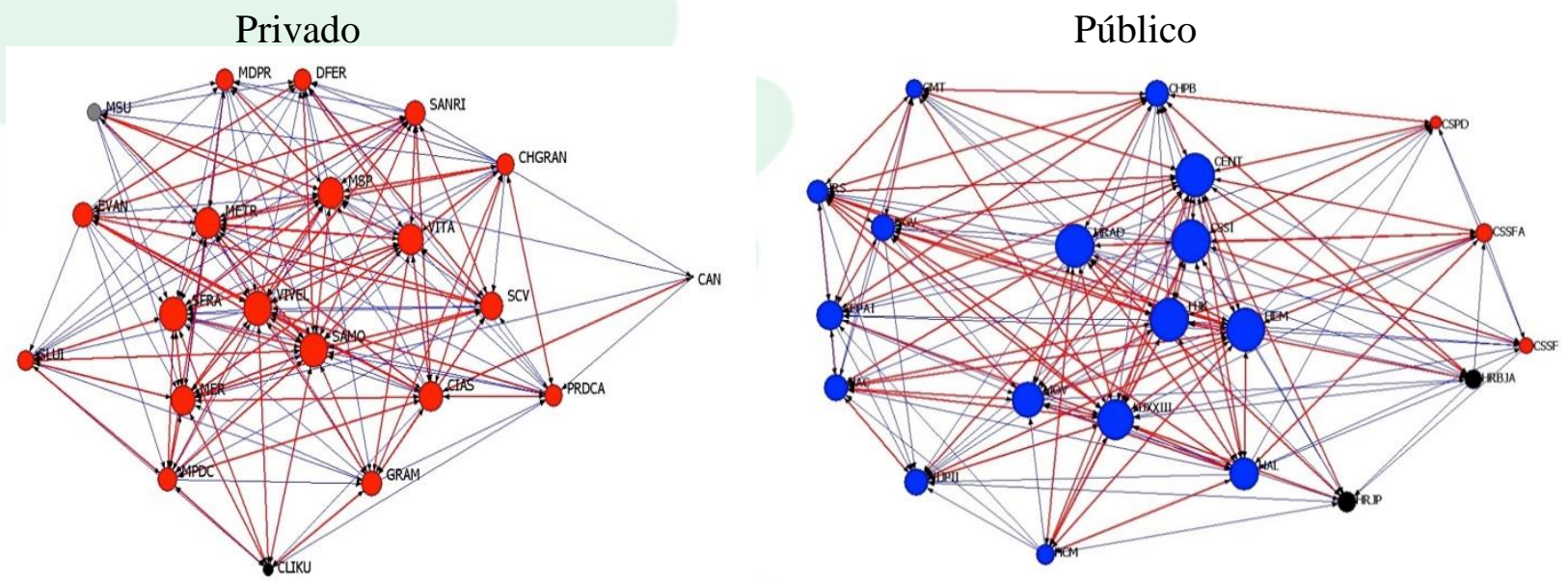

Fonte: Elaborado pelos autores.

Pode-se observar na Fig. 3. que os gráficos relacionais de atuação em rede do setor público e privado são similares. Em sua tipologia apresenta-se parcialmente descentralizado e bem denso com maioria enlaces (setas que ligam os nós) recíproco, destacado na cor vermelha, o que demonstra a forte relação entre os atores da rede, podendo inferir que seja uma característica do setor. Podendo ser um dos motivos que até mesmo os hospitais privados realizam atendimento pelo SUS, não havendo em si uma concorrência de mercado, diferenciando o segmento de serviços hospitalares dos demais. Apesar de similares, a rede pública possui relações recíprocas mais descentralizadas, isto se deve a institucionalização da rede. A FHEMIG, possui uma unidade central de administração que auxiliaas unidades hospitalares. Ao contrário da rede da RMGV de hospitais privados. Entretanto, por ser uma rede privada, a rede da RMGV se destaca pela conectividade dos seus atores. Algo a ser inferido sobre isso, é que os sindicatos patronais do setor de saúde são conhecidos pelo seu alto grau de corporativismo, natural da classe média de uma forma geral. A atuação em conselhos, associações podem ser usadas para criar barreiras de entradas (POTER, 1985) como diminuir as incertezas do ambiente externo.

Outro fator relevante é a quantidade de nós (hospitais) no centro e periferia da rede. Pode-se observar que a quantidade dos atores é bem parecida. Uma explicação para essas características é o perfil dos hospitais das duas redes, que mesmo sendo de outros estados e objetivo mercadológico o tipo de atendimento é bem diversificado nas duas populações, com maternidades, hospitais gerais, clinicas especializas entre outros. Uma característica observada é que os hospitais gerais especializados em pronto atendimento 
tendem a ficar no centro da rede, por atender as demandas até mesmo das outras unidades hospitalares.

No grapho relacional cooperação se originou da pergunta "Com quem o hospital COOPERA dentro da rede (troca recursos, troca informações, compartilha conhecimentos, possui projetos em comum)".
Cooperação é um dos pilares teóricos deste estudo, no caso, buscou-se captar como funciona a estrutura cooperativa das empresas por meio da rede. Para inferir sobre estratégias utilizadas, segue a apresentação gráfica na Fig. 4.

Figura 4 - Cooperação - Privado versus Público
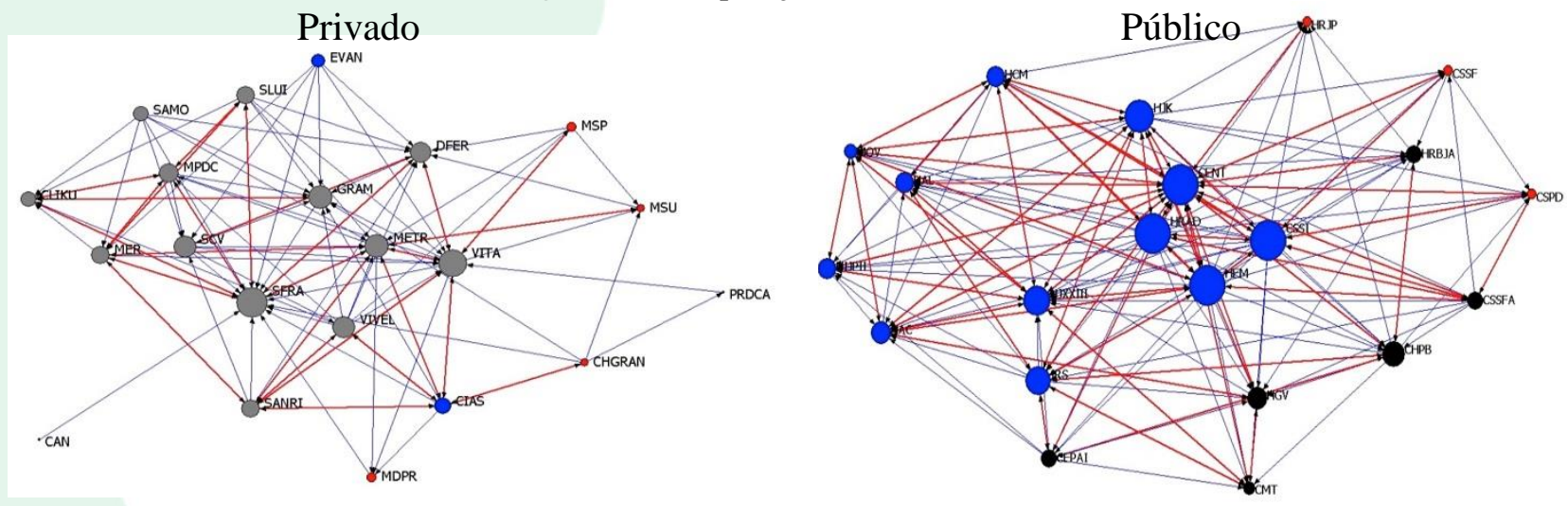

Fonte: Elaborado pelos autores.

A rede relacional Cooperação já apresenta mudanças significativas comparadas ao grapho relacional Atuação em Rede. A rede privada possui menor conectividade do que a rede pública, isto se deve a institucionalização da rede FHEMIG, que não só influência como modifica o caráter obrigatório da cooperação. Entretanto isso não necessariamente significa que a rede privada não possui alta cooperação. Para o ambiente privado e competitivo a rede da RMGV possui alta densidade de relações com muitas relações recíprocas. Entretanto, na rede privada alguns atores estão na periferia e de forma isolada, ligados à rede apenas por um laço não recíproco (setas de cor azul). Já na rede pública, todos os atores possuem pelo menos uma relação recíproca (setas de cor vermelha). Visualizando a tipologia da rede, a rede pública está mais descentralizada comparada a rede privada. Uma das pré-disposições dos atores do centro é o tamanho do escopo de recursos, que proporcionada uma vantagem competitiva.

Uma evidência empírica a ser observada no segmento hospitalar, comparando as duas realidades distantes (rede publica em Minas Gerais versus rede privada no Espírito Santo) é a forte atuação em rede. Pode-se inferir que o comportamento das organizações está evoluindo. Atuar em rede e cooperar já é uma estratégia utilizada neste segmento que tem em sua atuação especificidades e complexidades que exigem a redução de incertezas, o compartilhamento de recursos e desenvolvimento de conhecimentos.

O grapho relacional aprendizado (Fig. 5) se originou da pergunta "Com qual hospital vocês APRENDEM dentro da rede (pelo conhecimento tácito, pela experiência de mercado, pelos contatos, pela tecnologia desenvolvida)". Nesta pergunta buscou-se captar a percepção dos gestores sobre hospitais que conseguem repassar determinados conhecimentos relevantes para o setor. Como se observa no grapho relacional aprendizado na rede, a rede pública possui uma densidade de relações maior que a rede privada. Isto pode ser explicado pelo tempo que a rede existe possibilitando o acumulo do conhecimento relacional e desenvolvimento de novos processos que fomentam esse aprendizado. Entretanto, para uma rede privada, existe uma densidade significativa, mas as ligações recíprocas não alcançam todos os membros da periferia da rede. Enquanto na rede privada possuem diversos atores centralizados, na rede pública destacam quatro atores pelo tamanho e conectividade. 
Figura 5 - Aprendizado relacional - Privado versus Público

Privado

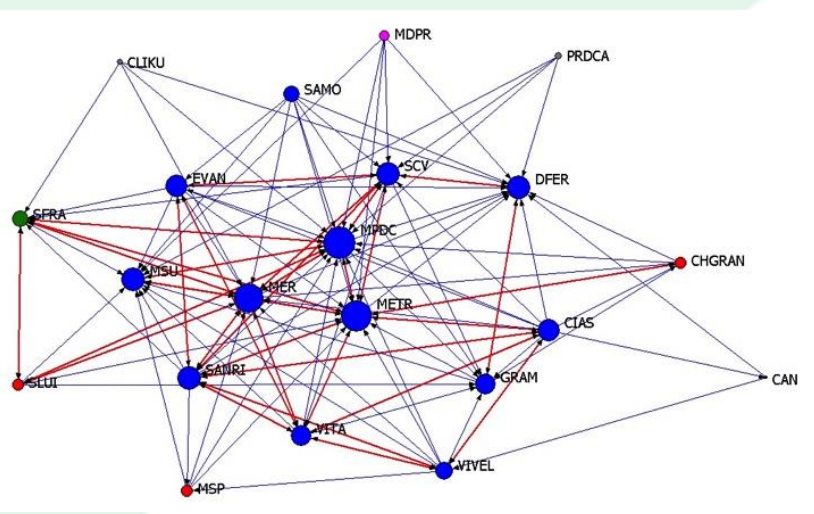

Público

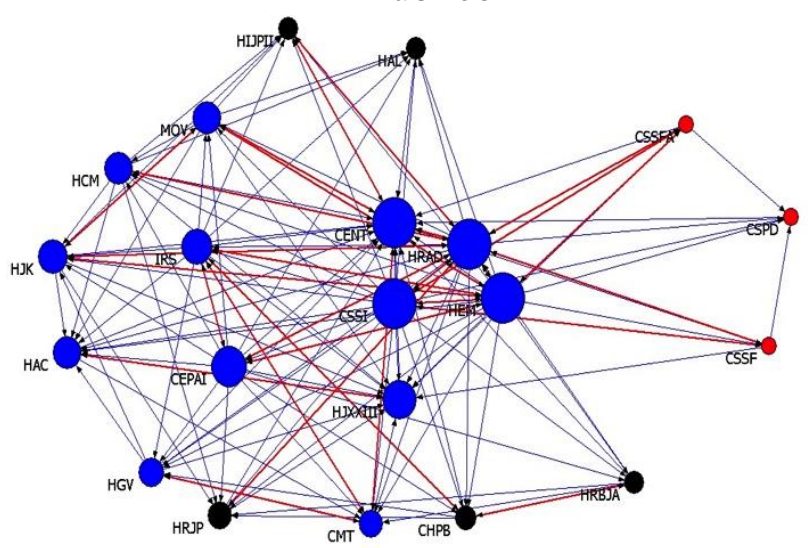

Fonte: Elaborado peıos autores.

Para melhor compreensão das análises de rede, têm-se (Fig. 6) alguns índices e graus que colaboram para interpretação dos resultados. A análise das medidas priorizou a centralidade (entrada) indegree e (saída) outdegree, medidas menos sensíveis para valores faltantes em grupos de dados (Balestrin, Verschoore, \& Perucia, 2014). Ao analisar o quadro geral das análises de redes, observa-se que tanto a pública quanto a privada possuem 21 hospitais neste estudo, possibilitando melhor ainda a comparação. As redes privadas de atuação em rede e aprendizado relacional obtiveram maior densidade do que as redes públicas. Entretanto, a rede pública possibilitou maior reciprocidade nas relações, com isso obteve maior grau de centralidade do que a rede privada. O grau de intermediação da rede pública foi menor nos graphos atuação em rede e cooperação e maior que a rede privada no grapho aprendizado relacional. O grau de proximidade da rede pública foi maior que o da rede privada, por consequência das relações recíprocas.

Com o objetivo de validar as hipóteses relacionadas ao modelo hipotético foram realizadas análises que verificaram a relação linear entre os construtos propostos na pesquisa. Primeiro, foram desenvolvidos gráficos de dispersão para todas as três hipóteses levantadas e foram calculados os respectivos coeficientes de correlação de Pearson. De acordo com Hair Jr, Anderson, Tatham e Black (2005), o coeficiente de correlação linear de Pearson é uma medida da força da relação entre duas variáveis que representam dados quantitativos. O coeficiente de correlação varia entre -1 e +1 , de modo que $-1 \leq \mathrm{r} \leq 1$, e quanto mais próximos desses extremos maior a relação linear entre as variáveis. Quanto mais próximo a zero maior o indício de que as variáveis não são correlacionadas. O que se deseja verificar é se a variável referente à um construto aumenta ou diminui em proporções parecidas quando outro construto se altera. Uma vez que as correlações lineares existam e sejam validadas por testes de hipótese, tem-se indícios sobre a causa e o efeito de uma variável em relação a outra. A Análise de Regressão foi utilizada para verificar essas relações. Somente após a análise estatística descritiva é que os modelos de regressão linear pelos métodos de mínimos quadrados foram ajustados.

Figura 6 - Índices de centralidade e coesão das redes - Público versus Privado

\begin{tabular}{|c|c|c|c|c|c|c|c|c|}
\hline \multirow{5}{*}{ 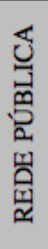 } & Medidas & \multicolumn{2}{|c|}{ Tamanho } & \multirow{2}{*}{$\begin{array}{c}\text { Densidade } \\
\text { (Normalizada\%) }\end{array}$} & \multirow{2}{*}{$\begin{array}{c}\text { Reciprocidade } \\
\text { (Normalizada\%) }\end{array}$} & \multirow{2}{*}{\begin{tabular}{|c|} 
Centralidade \\
(média do índice \\
normalizado \%)
\end{tabular}} & \multirow{2}{*}{$\begin{array}{c}\text { Intermediação } \\
\text { (média do índice } \\
\text { de centralidade) }\end{array}$} & \multirow{2}{*}{$\begin{array}{c}\text { Proximidade } \\
\text { (média do índice } \\
\text { de entrada) }\end{array}$} \\
\hline & Construtos & Atores & Relações & & & & & \\
\hline & Atuação em Rede & 21 & 244 & 58 & 56 & 58,09 & 2,2 & 72 \\
\hline & Cooperação & 21 & 203 & 48 & 62 & 48,33 & 2,71 & 66,95 \\
\hline & Aprendizado Relacional & 21 & 156 & 37 & 36 & 37,14 & 4,37 & 56,74 \\
\hline \multirow{5}{*}{ 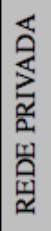 } & Medidas & \multicolumn{2}{|c|}{ Tamanho } & \multirow{2}{*}{$\begin{array}{c}\text { Densidade } \\
\text { (Normalizada\%) }\end{array}$} & \multirow{2}{*}{$\begin{array}{c}\text { Reciprocidade } \\
\text { (Normalizada\%) }\end{array}$} & \multirow{2}{*}{\begin{tabular}{|c} 
Centralidade \\
(média do índice \\
normalizado \%)
\end{tabular}} & \multirow{2}{*}{\begin{tabular}{|c} 
Intermediação \\
(média do índice \\
de centralidade)
\end{tabular}} & \multirow{2}{*}{$\begin{array}{l}\text { Proximidade } \\
\text { (média do índice } \\
\text { de entrada) }\end{array}$} \\
\hline & Construtos & Atores & Relações & & & & & \\
\hline & Atuação em Rede & 21 & 392 & 84 & 50,31 & 36,99 & 4,56 & 70,2 \\
\hline & Cooperação & 21 & 181 & 39 & 28,89 & 24,99 & 4,26 & 46,47 \\
\hline & Aprendizado Relacional & 21 & 226 & 48,9 & 30,56 & 30,07 & 3,16 & 46,1 \\
\hline
\end{tabular}

Fonte: Elaborado pelos autores. 
Todos os resultados foram significativos. Ressalta-se que, ao analisar o Beta padronizado (Fig. 7), as forças das relações entre os construtos "Atuação em Rede" e "Cooperação" (H1), e entre os construtos "Cooperação" e "Aprendizagem Relacional" (H2) foram maiores na rede privada do que na rede pública. Entretanto, a força da relação entre os construtos "Aprendizagem Relacional" e "Atuação em Rede" (H3) foi maior na rede publica do que na privada. Isto é, o reforço da atuação em rede é mais representativo na rede pública do que na privada e aprender na rede não significa aumentar a atuação, no caso da rede privada.

Procurando maiores evidências, foram realizados testes de hipóteses (Fig. 7) para verificar se é aconselhável ou não rejeitar a hipótese nula (H0) de que não há correlação linear entre os construtos, ou seja, o coeficiente de correlação de Pearson é igual a zero $(r=0)$. Rejeitar a hipótese nula é o mesmo que afirmar que a correlação entre dois construtos é estatisticamente significativa, portanto diferente de zero. De acordo com Hair Jr, Anderson, Tatham e Black (2005), ao se calcular o Valor P quando esse é menor ou igual ao nível de significância conclui-se que há uma correlação linear significativa entre as variáveis. Caso contrário, não existe evidência suficiente para apoiar a afirmação de que exista uma correlação linear. Segundo Hair Jr, Anderson, Tatham e Black (2005), o valor P é a probabilidade de se obter um valor da estatística de teste que seja, no mínimo, tão extremo quanto o que foi encontrado e representa os dados amostrais, supondo que a hipótese nula seja verdadeira. A hipótese nula é rejeitada se o valor $\mathrm{P}$ for muito pequeno, menor que o nível de significância do teste $(\alpha)$. Utilizando um nível de significância de 0,05 (5\%), não existe evidência estatística para rejeitar nenhuma hipótese. Com estes modelos de causa e efeito espera-se encontrar modelos de regressão lineares significativos para todas as hipóteses. Todos os resultados foram significativos.

A Regressão Linear, segundo Hair Jr, Anderson, Tatham e Black (2005) permite que seja identificada o tipo de relação estatística que existe entre uma variável dependente (ou explicativa) e uma variável independente (ou resposta). A Fig. 7 apresenta o resultado das principais medidas estatísticas dos modelos de Análise de Regressão ajustados. Os modelos foram gerados utilizando-se o software SPSS. As medidas estatísticas e suas interpretações permitem testar o modelo hipotético viabilizando a decisão de aceitar ou não as hipóteses formuladas. Para os modelos de regressão, os quais se verificaram a existência de relações lineares e a identificação de uma inclinação da reta de regressão pelo coeficiente $\beta 1$ foram realizados os testes de validação do modelo.

De acordo com Hair Jr, Anderson, Tatham e Black (2005) para a validação de um modelo de Regressão Linear é necessário que três suposições acerca dos resíduos ou erros de previsão sejam atendidas: os resíduos devem ser distribuídos segundo uma distribuição de probabilidade normal; os resíduos são independentemente distribuídos ou não correlacionados, de forma que o valor de um erro não depende de qualquer outro erro; os resíduos possuem variância constante, ou seja, o modelo seja homocedástico, de forma que a variância dos erros seja constante para qualquer valor da variável explicativa. A Fig. 7 apresenta as medidas estatísticas calculadas por intermédio da Regressão Linear para cada hipótese do modelo hipotético. A primeira delas é o r2 que segundo Hair Jr, Anderson, Tatham e Black (2005) mede a proporção da variação em y (variável dependente ou resposta) que pode ser explicada pela variável $\mathrm{x}$ (independente ou explicativa) no modelo de regressão. O r2 ajustado foi apresentado na Fig.7.

Figura 7 - Resultados da análise de regressão - Público versus Privado.

\begin{tabular}{|c|c|c|c|c|c|c|c|c|c|}
\hline \multirow{4}{*}{ 预总 } & Hipóteses & Modelos de Regressão & & $\beta$ & E.P. $(\beta)$ & Vaor-p & Bpadr. & $\mathrm{R}^{2}$ & $\mathrm{R}^{2}$ adj. \\
\hline & H1 & Atuação em Rede & Cooperação & 0,278 & 0,067 & 0,001 & 0,688 & 0,473 & 0,44526 \\
\hline & $\mathrm{H} 2$ & ção & Apr & 0,778 & 0,179 & 0,000 & 0,706 & 0,499 & 0,472 \\
\hline & $\mathrm{H} 3$ & do & em Rede & 0,371 & 0,056 & 0,000 & 0,833 & 0,649 & 0,678 \\
\hline \multirow{4}{*}{ 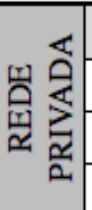 } & Hipóteses & Modelos de Regressão & Variável (Y) & $\beta$ & E.P. $(\beta)$ & Vaor-p & Bpadr. & $\mathrm{R}^{2}$ & $\mathrm{R}^{2}$ adj. \\
\hline & H1 & & & 0,530 & 0,108 & 0,000 & 0,741 & 0,549 & 0,526 \\
\hline & $\mathrm{H} 2$ & Cooperação & Aprendizado & 0,920 & 0,165 & 0,000 & 0,782 & 0,612 & 0,526 \\
\hline & $\mathrm{H} 3$ & Aprendizado & Atuação em Rede & 0,592 & 0,137 & 0,000 & 0,694 & 0,481 & 0,453 \\
\hline
\end{tabular}

Fonte: Elaborado pelos autores. 


\section{CONCLUSÃO}

Neste estudo foi possível alcançar o objetivo proposto de compreender como os fatores relacionais (i) Atuação em Rede; (ii) Cooperação; e (iii) Aprendizagem Relacional - se desenvolvem em comparação as redes hospitalares público x privado, de MG e ES. Observou-se que todos os resultados foram significativos. Destaca-se a existência de diferenças pontuais em relação a fatores relacionais entre as redes analisadas. As hipóteses $\mathrm{H} 1$ e $\mathrm{H} 2$ da rede privada foram mais representativas que da rede pública, enquanto a $\mathrm{H} 3$ da rede publica foi mais representativa que da rede privada. Isto é, na rede privada considerase que atuar em rede conduz à cooperação, bem como a cooperação conduz ao aprendizado. Porém, atuar em rede, cooperar e aprender, não significa, necessariamente, aumentar a atuação em rede. Em paralelo, na rede pública, observa-se maior relação entre a aprendizagem relacional e o reforço da atuação em rede. Além disso, observa-se que a rede pública possui maior reciprocidade nas relações e, por isso, possibilita de forma geral uma maior proximidade dos seus atores.

Observa-se nos resultados que as redes hospitalares públicas e privada possui uma forte tendência que cooperar leva ao aprendizado. Entretanto, como argumentado por Borgatti \& Foster, (2003) o a vontade de aprender pode em breve desaparecer à medida que os atores se esforçam pouco para utilizar as soluções tecnológicas para ajudar as organizações a armazenar, compartilhar e criar novos conhecimentos, ou seja, à medida que os atores percebem que não estão aprendendo quanto desejam, pode-se criar buracos estruturais. Outro ponto relevante é se este conhecimento produzido pelo processo de aprendizado não é inovador, pode-se influenciar a redução de laços para busca de novos atores para oxigenar a rede.

Dentre as limitações deste estudo, destacamse: (1) impossibilidade de se deduzir padrões aplicáveis a uma população de redes alheia ao objeto de estudo, imposta pelo próprio método utilizado; (2) número de respondentes reduzidos à 21 por rede, o que influencia negativamente a análise de regressão linear realizada; e (3) amostra por conveniência para análise estatística. Apesar disso, este estudo foi importante para aprofundar a compreensão e diferença das redes hospitalares público e privado. Este estudo abre margem para novas questões, como: verificar as premissas do modelo em outro contexto de redes; compreender o porquê das redes públicas tenderem a reforçar sua atuação em rede quando percebem estar aprendendo com a interação relacional, enquanto a rede privada não; entre outras que possibilitem novas compreensões na atuação organizacional em rede.

\section{REFERÊNCIAS}

Ahuja, G. (2000). Collaboration networks, structural holes, and innovation: A longitudinal study. Administrative science quarterly, 45(3), 425-455.

Amato Neto, J. (2000). Redes de cooperação produtiva e clusters regionais: oportunidades para as pequenas e médias empresas. Editora Atlas.

Azevedo, T. B. D., \& Rodriguez, M. V. R. Y. (2010). Softwares para análise de redes sociais-ARS. In VI Congresso Nacional de Excelência em Gestão. Niterói.

Balestrin, A., \& Fayard, P. (2003). Redes interorganizacionais como espaço de criação de conhecimento. XXVII Encontro da Associação Nacional de Pós-Graduação e Pesquisa em Administração. Anais, Atibaia: ANPAD.

Balestrin, A., Verschoore, J. R., \& Junior, E. R. (2010). O Campo de Estudo sobre Redes de Cooperação Interorganizacional no Brasil. Revista de Administração Contemporânea, 14(3), 459.

Balestrin, A., Verschoore, J. R., \& Perucia, A. (2014). A Visão Relacional da Estratégia: Evidências Empíricas em Redes de Cooperação Empresarial. BASE-Revista de Administração e Contabilidade da Unisinos,11(1), 47-58.

Barringer, B. R., \& Harrison, J. S. (2000). Walking a tightrope: Creating value through interorganizational relationships. Journal of management, 26(3), 367-403.

Borgatti, S. P. (2002). NetDraw: Graph visualization software. Harvard: Analytic Technologies.

Borgatti, S. P., \& Foster, P. C. (2003). The network paradigm in organizational research: A review and typology. Journal of management, 29(6), 991-1013.

Brasil. Ministério da Saúde. (2011) Cadastro Nacional de estabelecimento de saúde. <http://tabnet.datasus.gov.br/cgi/cnes/NT_Estabele cimentos.htm> Acesso em: 13 de jul. 2001b.

Brasil. Ministério da Saúde. (2009). O SUS de A a Z: garantindo saúde nos municípios, Conselho Nacional das Secretarias Municipais de Saúde, 3. ed. Brasília: Ed. do Ministério da Saúde.

Burt, R. S. (2009). Structural holes: The social structure of competition. Harvard university press. 
Carrington, P. J., Scott, J., \& Wasserman, S. (Eds.). (2005). Models and methods in social network analysis (Vol. 28). Cambridge university press.

Castells, M. (1999). A sociedade em rede, vol. 1. São Paulo: Paz e Terra.

Daft, R. L. (2006). Administração. Pioneira Thomson Learning.

De Borba, G. S., \& Neto, F. J. K. (2008). Gestão Hospitalar: identificação das práticas de aprendizagem existentes em hospitais. Saúde e Sociedade, 17(1), 44-60.

Drucker, P. F. (1999). Sociedade pós-capitalista. Rio de Janeiro.

Espirito Santo (Estado). (2005). Lei complementar $\mathrm{n}^{\mathrm{o}}$ 318 , de 17 janeiro de 2005. Reestrutura a Região Metropolitana da Grande Vitória - RMGV. Diário Oficial do Espírito Santo.

Fleury, A. C. C., \& Fleury, M. T. L. (1997). Aprendizagem e inovação organizacional: as experiências de Japão, Coréia e Brasil. Atlas.

Freeman, L. (2004). The development of social network analysis. A Study in the Sociology of Science.

Freeman, L. C. (1996). Some antecedents of social network analysis.Connections, 19(1), 39-42.

Glouberman, S., \& Mintzberg, H. (2001). Managing the care of health and the cure of disease-Part I: Differentiation. Health care management review,26(1), 56-69.

Gonçalves, C. A., \& de Moraes Meirelles, A. (2004). Projetos e relatórios de pesquisa em administração. Atlas.

Gonçalves, C. A., Lebarcky, F. V., \& Muylder, C. F. (2015). As Cinco Forças Amizade, Contato, Aprendizado, Cooperação e Competição como Campo de Força Estratégica de Redes: um estudo no setor de calçados e bolsas. Revista de Ciências da Administração, 17(42), 38.

Granovetter, M. S. (1973). The strength of weak ties. American journal of sociology, 1360-1380.

Gulati, R., \& Gargiulo, M. (1999). Where do interorganizational networks come from? 1. American journal of sociology, 104(5), 1439-1493.

Hair Junior, J. F., Anderson, R. E., Tatham, R. L., \& Black, W. C. (2005). Análise multivariada de dados.
Hitt, M. A., Ireland, R. D., \& Hoskisson, R. E. (2008). Administração estratégica. Pioneira Thomson Learning.

Jarillo, J. C. (1988). On strategic networks. Strategic management journal,9(1), 31-41.

La Forgia, G. M., \& Couttolenc, B. F. (2009). Desempenho hospitalar no Brasil. São Paulo: Singular.

Lago Júnior, M. W. D. (2005). Redes sociais informais intraorganizacionais e os processos de mudanças organizacionais: Estudo em uma empresa de tecnologia da informação.

Lazzarini, S. G. (2008). Empresas em rede. São Paulo: Cengage Learning, 17-34.

Malhotra, N. K. (2012). Pesquisa de marketing: uma orientação aplicada. Bookman Editora.

Marsden, P. V. (2005). Recent developments in network measurement.Models and methods in social network analysis, 8, 30 .

Migueletto, D. C. (2001). Organizações em rede. Rio de Janeiro, 96.

Nonaka, I., \& Takeuchi, H. (1997). Criação de conhecimento na empresa: como as empresas japonesas geram a dinâmica da inovação. Rio de Janeiro. Campus, 16, 360.

Owen-Smith, J., \& Powell, W. W. (2004). Knowledge networks as channels and conduits: The effects of spillovers in the Boston biotechnology community. Organization science, 15(1), 5-21.

Peci, A. (1999). Emergência e proliferação de redes organizacionais: marcando mudanças no mundo de negócios. Revista de Administração Pública, 33(6), 7-24.

Phelps, C., Heidl, R., \& Wadhwa, A. (2012). Knowledge, networks, and knowledge networks a review and research agenda. Journal of Management, 38(4), 1115-1166.

Porter, M. E., \& Teisberg, E. O. (2007). Repensando a saúde. Bookman.

Porter, Michael E. "Cluster and the new economics of competition." (1998).

Prahalad, C. K., \& Hamel, G. (2005). Competindo pelo futuro: estratégias inovadoras para obter o controle do seu setor e criar os mercados de amanhã. Gulf Professional Publishing. 
Provan, K. G., \& Milward, H. B. (1995). A preliminary theory of interorganizational network effectiveness: A comparative study of four community mental health systems. Administrative science quarterly, 133.

Provan, K. G., Fish, A., \& Sydow, J. (2007). Interorganizational networks at the network level: A review of the empirical literature on whole networks.Journal of management, 33(3), 479-516.

Ring, P. S., \& Van de Ven, A. H. (1994). Developmental processes of cooperative interorganizational relationships. Academy of management review, 19(1), 90-118.

Santos, A. C. (1998). Análise Setorial: Sistema Hospitalar. São Paulo: Gazeta Mercantil.

Scott, J. (2012). Social network analysis. Sage.

Senge, P. M. (2009). A quinta disciplina: arte e prática da organização que aprende. Best Seller.
Shortell, S. M., \& Kaluzny, A. D. (2000). Health care management: organization, design, and behavior. Cengage Learning.

Smith, K. G., Carroll, S. J., \& Ashford, S. J. (1995). Intra-and interorganizational cooperation: Toward a research agenda. Academy of Management journal, 38(1), 7-23

Vasconcellos, G. M. V., \& Lopes, H. E. G. (2010). Cooperação e Alianças: Perspectivas Teóricas e suas Articulações no Contexto do Pensamento Estratégico/Alliances and Cooperation: Theoretical Outlooks in the Realm of Strategic Thought. Revista de Administração Contemporânea, 14(4), 722.

Verschoore, J. R. (2004). Redes de cooperação: uma nova organização de pequenas e médias empresas no Rio Grande do Sul. Porto Alegre: FEE, 1-46.

Wasserman, S., \& Faust, K. (1994). Social network analysis: Methods and applications (Vol. 8). Cambridge university 